\title{
Multicomponent Materials to Improve Solubility: Eutectics of Drug Aminoglutethimide
}

Basanta Saikia, Andreas Seidel-Morgenstern and Heike Lorenz Max Planck Institute for Dynamics of Complex Technical Systems, Magdeburg, Germany E-mail: bsaikia1@gmail.com, saikia@mpi-magdeburg.mpg.de

\begin{abstract}
Here, we report the synthesis and experimental characterization of three drug-drug eutectic mixtures of drug aminoglutethimide (AMG) with caffeine (CAF), nicotinamide (NIC) and ethenzamide (ZMD). The eutectic mixtures (AMG-CAF, AMG-NIC and AMG-ZMD) demonstrate significant melting point depressions ranging from 99.2 to $127.2{ }^{\circ} \mathrm{C}$ compared to the melting point of the drug $\mathrm{AMG}\left(151^{\circ} \mathrm{C}\right)$ and also show significantly higher aqueous solubilities than that of the AMG. The results presented include the determination of the binary melt phase diagrams and accompanying analytical characterization via X-ray powder diffraction, FT-IR spectroscopy and Scanning electron microscopy.
\end{abstract}

\section{Introduction}

The multicomponent solid form of the drug is rapidly emerging as an effective way to improve the drug physiochemical properties such as solubility, dissolution rate, bioavailability and other crucial pharmaceutical properties like stability, hygroscopicity, chemical stability, flowability etc. ${ }^{1-7}$ Eutectic mixtures are multicomponent compounds made up of two or more crystalline solids that show immiscibility in the solid-state and do not combine to generate a new chemical compound but, at a certain ratio, the eutectic composition, exhibit a melting or solidification point significantly lower than its constituents. ${ }^{1,4,8}$ The formation of eutectic mixtures can occur via different noncovalent interactions primarily hydrogen bonding, van der Waals forces, and aromatic interactions etc. ${ }^{9-10}$ It is a trial and error approach to get a eutectic composition for enhancing the solubility and bioavailability of medicines with limited water solubility in BCS classes II and IV. ${ }^{1,4}$ Eutectic mixtures are frequently used for the design of medicines and delivery methods for administration routes. ${ }^{1,11}$ They generally comprise with high thermodynamic parameters, for example, free energy, enthalpy and entropy etc., which alter the solubility and dissolution behaviour. ${ }^{1}$ Besides, a higher soluble eutectic mixture component, called coformer in the 
following, also can favourably influence the wettability of the drug, thus improving the bioavailability. ${ }^{12-13}$ When taken orally, curcumin, for example, has low bioavailability and solubility. However, the eutectic mixture of curcumin and nicotinamide in a 1:2 ratio exhibits a 10-fold faster intrinsic dissolution rate. ${ }^{14}$ Another aspect is that it is critical to identify the production of eutectics during the formulation stage to minimize manufacturing difficulties. ${ }^{15-16}$ During pharmaceutical research, understanding eutectic mixtures can aid in the discovery of compounds with equivalent melting points.

In this work, we studied the chiral drug aminoglutethimide [3-(4-aminophenyl)-3-ethyl-2, 6piperidinedione] (AMG), a nonsteroidal aromatase inhibitor drug, used for the treatment of Cushing's syndrome, breast cancer, and prostate cancer. ${ }^{17-19}$ According to the Biopharmaceutics Classification System (BCS), AMG is classified as a BCS class II drug because of its poor solubility. ${ }^{20}$ Attempts were made in this work to create multidrug eutectics of AMG considering caffeine (CAF, a psychoactive drug ${ }^{21}$ ), nicotinamide (NIC, a vitamin ${ }^{22}$ ) and ethenzamide (ZMD, nonsteroidal anti-inflammatory $\mathrm{drug}^{23}$ ) as coformers to improve the aqueous solubility of AMG. Scheme 1 shows the chemical structures of AMG and the three coformers. For the synthesis of eutectics mainly a mechanochemical solvent-assisted grinding (LAG) method has been used. Differential scanning calorimetry (DSC) data is utilized to establish the precise eutectic composition. X-ray powder diffraction (PXRD), Fourier-transform infrared spectroscopy (FT-IR), and Scanning electron microscopy (SEM) were applied to characterize the eutectic mixtures and their components. Further, the aqueous solubility of the eutectics was determined and found to significantly exceed that of the parent compound AMG.

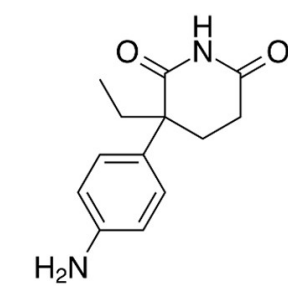

Aminoglutethimide (AMG)<smiles>NC(=O)c1cccnc1</smiles>

Nicotinamide

(NIC)<smiles>Cn1c(=O)c2c(ncn2C)n(C)c1=O</smiles>

Caffeine

(CAF)<smiles>CCOc1ccccc1C(N)=O</smiles>

Ethenzamide (ZMD)

Scheme 1 Molecular structure of Aminoglutethimide and the coformers selected for the study. 


\section{Materials and Methods}

\subsection{Materials}

Aminoglutethimide (purity: $>98.0 \%$ ) was purchased from TCI, Japan. Caffeine (purity: $>98 \%$ ), nicotinamide (purity: $>98 \%$ ) and ethenzamide (purity: $>97 \%$ ) were purchased from Sigma Aldrich (Germany). Millipore water from the Milli-Q system (Merck Millipore, Milli-Q Advantage) was used for solubility determination and HPLC-grade solvents for the mechanochemical experiments.

\subsection{Aminoglutethimide Eutectic Mixture Screening and Eutectic Composition Determination}

Multiple mixtures of AMG and the chosen coformer in different weight percentages, e.g. 50\%, $55 \%, 60 \%, 65 \%, 70 \%, 75 \%$, and $80 \%$, were prepared. The needed amount of each component was added to a mortar to obtain $100 \mathrm{mg}$ of the desired binary combination and grinded using mortar and pestle with dropwise addition of acetonitrile for 30 minutes.

The melting behavior of the resulting solids and the eutectic formation was determined by means of a DSC linear heating run. Eutectic and pure compounds melting temperatures were considered from the corresponding peak onsets, liquidus temperatures for mixtures were taken from the peak maximum. Therewith, the binary phase diagrams and corresponding Tammann plots were constructed to specify the eutectic composition of the respective drug-drug system. For the Tammann plot, the enthalpy of fusion of the eutectic melting effects in the DSC curves of the mixtures are used.

The melting of the eutectic mixture is characterized by a single melt peak in the DSC curve for the corresponding drug-drug, that is eutectic composition. This is based on the fact that the eutectic is an invariant point in the binary system in analogy to the melting point of a pure compound in a unary system

\subsection{Preparation of Bulk Mixtures at the Eutectic}

For liquid-assisted grinding, the components of each system were mixed and homogenized for 30 minutes in a glass mortar and pestle with acetonitrile as the solvent. The AMG-CAF, AMG-NIC and AMG-ZMD systems were produced in their respective eutectic compositions to perform solidstate characterization and solubility tests. In a nutshell, exact weights of AMG-CAF (75 wt. \% of AMG), AMG-NIC (50 wt. \% of AMG), and AMG-ZMD (50 wt. \% of AMG) systems were considered for the solubility evaluation. 


\subsection{Analytical Techniques}

Powder X-ray Diffraction (PXRD) The solid-state properties of the system were investigated by powder X-ray diffraction (PXRD) analysis with an X'pert Pro Diffractometer (PANalytical $\mathrm{GmbH}$, Kassel, Germany) with $\mathrm{Cu} \mathrm{K} \alpha$ radiation and an $\mathrm{X}$ 'Celerator detector in the 2-theta range of $3-40^{\circ}$ with a step size of $0.017^{\circ}$ and a step time of $50 \mathrm{~s}$.

Differential Scanning Calorimetry Thermal analysis was carried out using a DSC 131 (Setaram, Diepholz, Germany), which was regularly calibrated using highly pure standard materials. The DSC measurements were carried out in aluminium crucibles with a constant heating rate of 2 $\mathrm{K} / \mathrm{min}$, under a pure helium atmosphere at $8 \mathrm{~mL} / \mathrm{min}$.

Fourier Transform Infrared Spectroscopy (FT-IR). A Bruker ALPHA II FT-IR (Bruker, Karlsruhe, Germany) with diamond attenuated total reflectance (ATR) accessory was used to gather Fourier transform infrared spectra. The solid materials were placed in the ATR cell without further preparation and investigated in the $4000-400 \mathrm{~cm}^{-1}$ range, collecting $32 \mathrm{scans}$ at a resolution of $2 \mathrm{~cm}^{-1}$.

Scanning Electron Microscopy Scanning electron microscopy (SEM) was carried out by using an XL 30 FEG (FEI, Hilsoro, OR, USA) instrument at an acceleration voltage of $5 \mathrm{keV}$ and a working distance of $12.4 \mathrm{~mm}$.

\subsection{Solubility Determination}

The gravimetric method was used to determine the solubility of AMG and its binary eutectic mixtures in water at room temperature $\left(25^{\circ} \mathrm{C}\right)$. An excess amount of solid eutectic mixture was added to $4 \mathrm{~mL}$ water, and the suspension was stirred at $300 \mathrm{rpm}$ at $25^{\circ} \mathrm{C} \pm 1{ }^{\circ} \mathrm{C}$ for $72 \mathrm{~h}$ to reach equilibrium. Then it was allowed to settle before liquid phase sampling. The saturated solutions of respective eutectic mixtures were filtered using a $0.45 \mu \mathrm{m}$ syringe filter and transported in a $5 \mathrm{~mL}$ vial. The weight of the full vial with the solution was recorded immediately. The vials were kept in a fume hood for evaporation of the water. Solids were obtained after $\sim 3-4$ days. They are further placed in a desiccator for 1 day. All measurements were carried out in duplicate. The solubility of pure AMG $(\mathrm{mg} / \mathrm{mL})$ in water was calculated from the product of the mass of the dried material (solubility of eutectic mixture) and the respective AMG content in the dried eutectic mixture (in weight fraction). 


\section{Results and Discussion}

In literature, it was pointed out ${ }^{1,10}$ that organic eutectic formation happens when the molecular interaction among identical molecules are comparably stronger than the interaction between different molecules. Till now, there are no exact rules to design eutectics based on the cohesive interactions dominant over the adhesive interaction to produce a eutectic. ${ }^{4,10}$ Generally, a cocrystal is expected to be obtained when the possibility of formation of very strong adhesive interactions is high. However, when the cohesive interactions are strong but auxiliary interactions are weak to nil, as long as molecular mismatched shapes are there, then the formation of a eutectic mixture is expected. ${ }^{10}$ In the case of $A M G$, the interactions like imide $\cdots$ imide and $\mathrm{N}-\mathrm{H} \cdots \mathrm{O}$ hydrogen bond interactions are relatively stronger (Figure 1). However, in the case of multicomponent systems with the coformers CAF, NIC and ZMD the adhesive interactions are relatively weaker as they are non-isomorphous molecules with a considerable mismatch in size and shape. Moreover, the structural arrangement will have lack a unique lattice arrangement distinct from the individual components and thus retains the cohesive interactions in its eutectic mixtures. Therefore, the Xray diffraction pattern and spectroscopic signature peaks of a eutectic mixture does not contain new crystalline arrangement, rather contains the overlapping of diffraction peaks of the individual components.
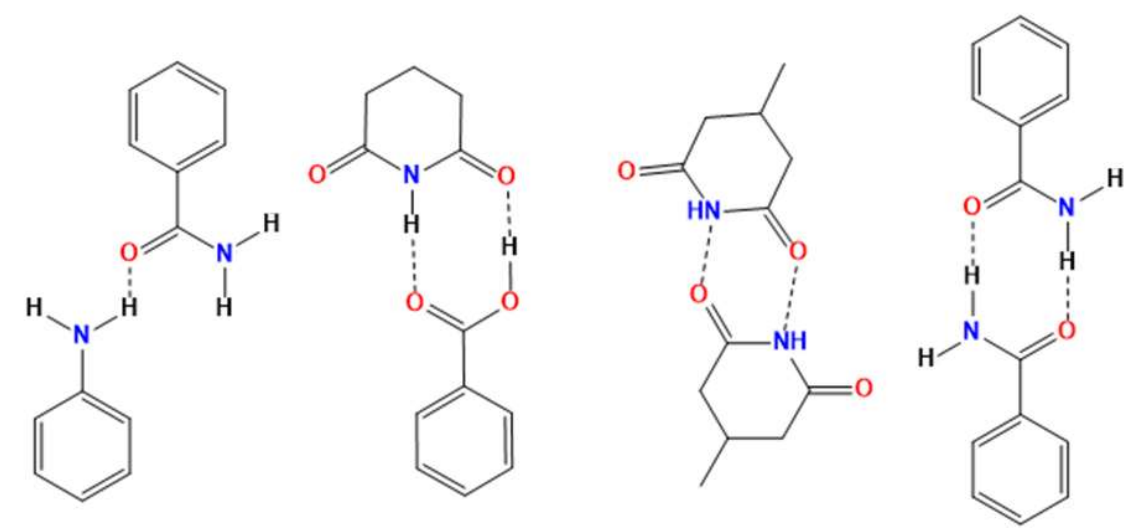

Figure 1 Examples of some probable supramolecular synthons that may be present in AMGeutectic systems.

The Cambridge Structural Database (CSD) search for imide and carboxylic acid interaction only reveals two reported crystal structures with refcode HUVGAI and UGOHUV, demonstrating the weaker probability of cocrystal formation via the imide $\cdots$ carboxylic acid heterosynthon. Similarly, no imide and only one monosubstituted pyridine interaction have been observed from 
CSD search for the multicomponent crystalline systems of imide functional molecules. There is also no reported structure with imide $\cdots$ carboxamide interaction. Furthermore, cocrystal screening by considering the molecular complimentary screening wizard of $\mathrm{CSD}^{24}$ also reveals no-hit for cocrystals for the selected coformers.

\section{Powder X-ray Diffraction (PXRD) Analysis}

$\mathrm{X}$-ray diffraction patterns can be used as one of the confirmatory tools for the detection of the formation of a eutectic system. Figure 2 displays the comparison between the PXRD patterns obtained for starting components and their respective eutectic systems.

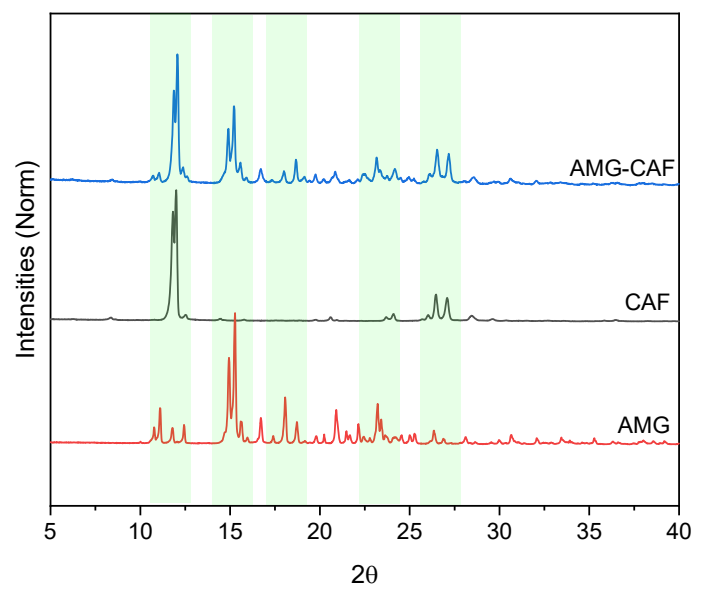

(a)

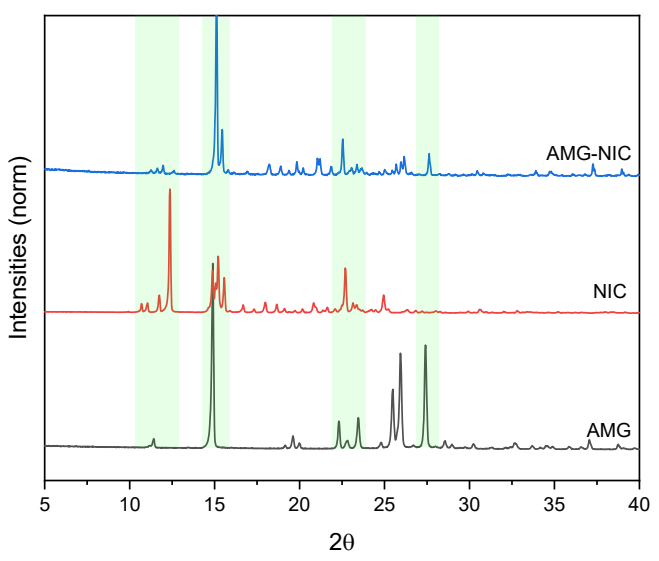

(b)

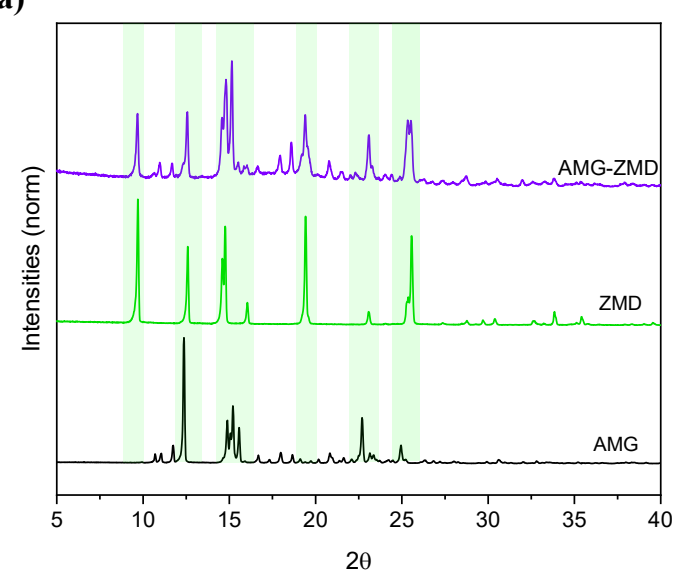

(c)

Figure 2: Overlay of PXRD patterns of (a) AMG, CAF and the eutectic mixture of the AMGCAF system, (b) AMG, NIC and the eutectic mixture of the AMG-NIC system and (c) AMG, ZMD and the eutectic mixture of the AMG-AMD system. Main characteristic peaks are indicated by dashes lines. 
In a eutectic system, the retention of the crystal structure of individual components is expected, consequently, the PXRD pattern of the eutectic system should contain the overlap of diffraction peaks of starting components without the generation of new diffraction peaks. As seen in the comparison of the diffraction patterns of the eutectic mixtures and the pure starting components, no new peaks were observed and all the diffraction peaks of the starting components are present in the grinded mixtures, signifying the formation of eutectic mixtures in all three studied cases.

\section{Differential Scanning Calorimetry (DSC) Analysis}

DSC is one essential technique to characterize the solid phase behavior of a material and also mixtures. It is crucial to detect the eutectic nature of a multicomponent system as typically the melting point of the eutectic mixture is lower than either of the parent components and the eutectic melting can be identified via the melting events occurring. The DSC patterns of the various AMGcoformer mixtures overlayed with those of the pure components are shown in Figure 3, specifically for the AMG-CAF system in Figure 3a, the AMG-NIC system in Figure 3b and the AMG-ZMD system in Figure 3c.

The thermogram of AMG exhibits only one endothermic melting peak, suggesting no polymorphic transition during the heating cycle. The eutectic melting peak for the AMG-NIC system (Figure 3 b) is found at $99.2^{\circ} \mathrm{C}$, being, as expected, significantly lower than for both the starting materials. A close-to single eutectic peak is observed for the appr 50\% mixture, indicating the presence of the "purely" eutectic phase and thus, the eutectic composition in the AMG-NIC system. In the case of the AMG-CAF system at different weight \% ratios, except at the eutectic composition, DSC curves exhibited three endothermic transition effects (see Figure 3a). The first endothermic peak signifies the eutectic temperature, whereas the second and third endotherms signify the melting of excess AMG and CAF respectively. The supplementary peak observed at $137.7^{\circ} \mathrm{C}$ corresponds to the enantiotropic phase transition of caffeine polymorphic form II to form I. ${ }^{25-26}$ The eutectic composition is found to be close to $75 \mathrm{wt}$ \% AMG composition. For the AMG-ZMD system, the melting endothermic peak at $101.8{ }^{\circ} \mathrm{C}$ refers to the eutectic melting (see Figure $3 \mathrm{c}$ ), while the eutectic composition is found close to the AMG-ZMD composition of $\sim 50$ wt. \%. Table 1 contains the measured temperatures and enthalpies of melting for the pure AMG, the coformers used and their eutectic mixtures. 


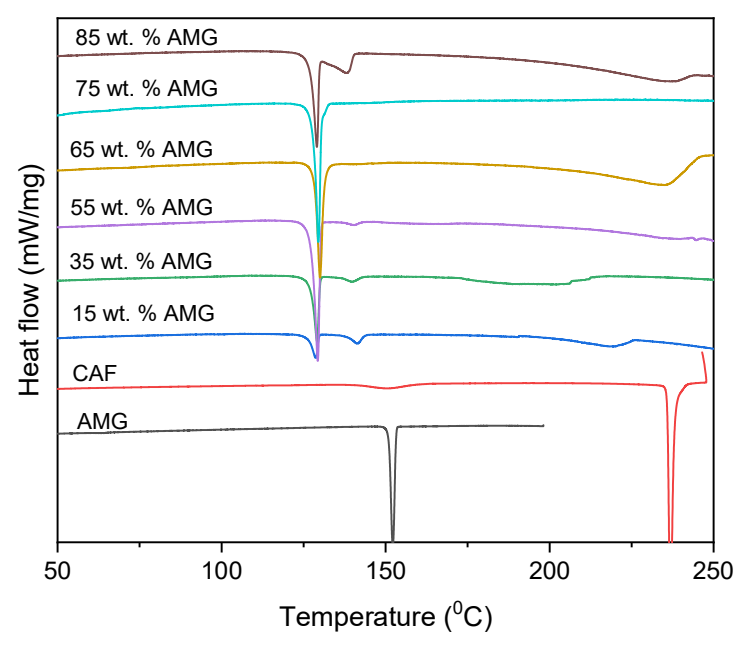

(a)

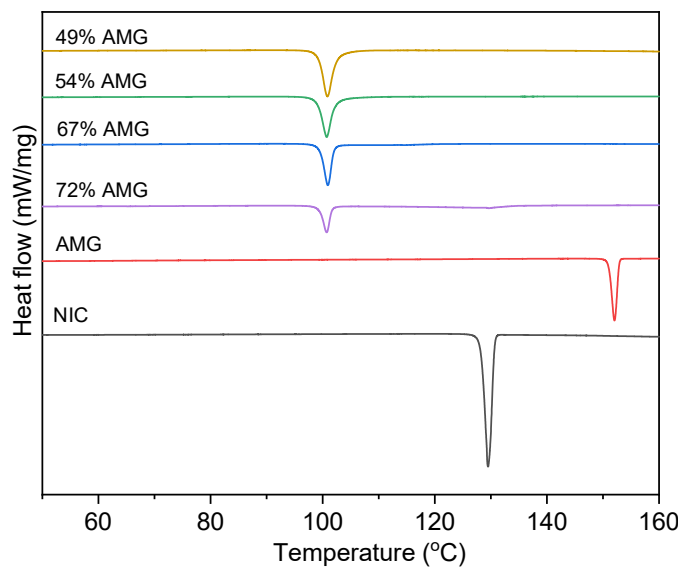

(b)

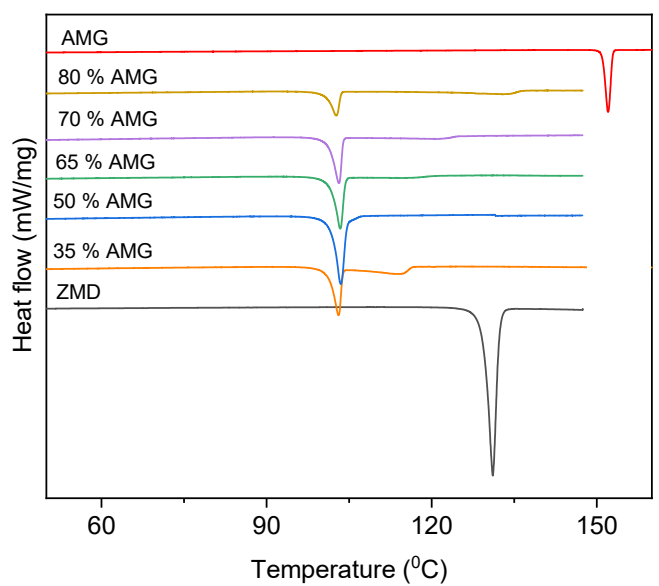

(c)

Figure 3 DSC patterns of the different AMG-coformer compositions showing the melting point depressions and the eutectic peak, (a) AMG and CAF, (b) AMG and NIC, and (c) AMG and ZMD.

Table 1 Melting point (M.P) and melting enthalpy (M.E) of drug AMG, the coformers and their eutectic compositions.

\begin{tabular}{lcccccc}
\hline Drug & Coformer & $\begin{array}{l}\text { M.P } \\
\left({ }^{\circ} \mathbf{C}\right)\end{array}$ & $\begin{array}{l}\text { M.E } \\
(\mathbf{J} / \mathbf{g})\end{array}$ & Eutectic & $\begin{array}{l}\text { M.P } \\
\left({ }^{\circ} \mathbf{C}\right)\end{array}$ & $\begin{array}{l}\text { M.E } \\
(\mathbf{J} / \mathbf{g})\end{array}$ \\
\hline AMG & CAF & 235.9 & 128.51 & AMG-CAF & 127.2 & 74.21 \\
\cline { 2 - 7 } M.P. $=151.0\left({ }^{\circ} \mathrm{C}\right)$ & NIC & 128.68 & 186.81 & AMG-NIC & 99.2 & 125.55 \\
\cline { 2 - 7 } M.E $=110.23 \mathrm{~J} / \mathrm{g}$ & ZMD & 129.4 & 174.7 & AMG-ZMD & 101.8 & 124.15 \\
\hline
\end{tabular}




\section{Infrared spectroscopy}

IR spectroscopy was used to understand the probable intermolecular interactions present in the eutectic system. The observed IR spectra of AMG, the coformers and the respective eutectic systems are presented in Figure 4. As seen, the IR spectra of the eutectic systems are comparable with their starting components. No new peak was observed in the three eutectic systems and the spectra simply overlap with the spectra of the individual components. All the characteristic vibrational bands of $\mathrm{AMG}$ and the coformers are observed in the spectrum of the eutectic composition. For the AMG spectrum, the $\mathrm{N}-\mathrm{H}$ starching peak is observed at $3470 \mathrm{~cm}^{-1}$, the $\mathrm{C}=\mathrm{O}$ stretch vibration is observed at $1713 \mathrm{~cm}^{-1}$. The slight shifting of $\mathrm{C}=\mathrm{O}$ peaks in the mixtures absorption bands around $1713-15 \mathrm{~cm}^{-1}$ might be caused by the long-range interaction between the carbonyl groups and other functionality in the molecules in the eutectic mixture.

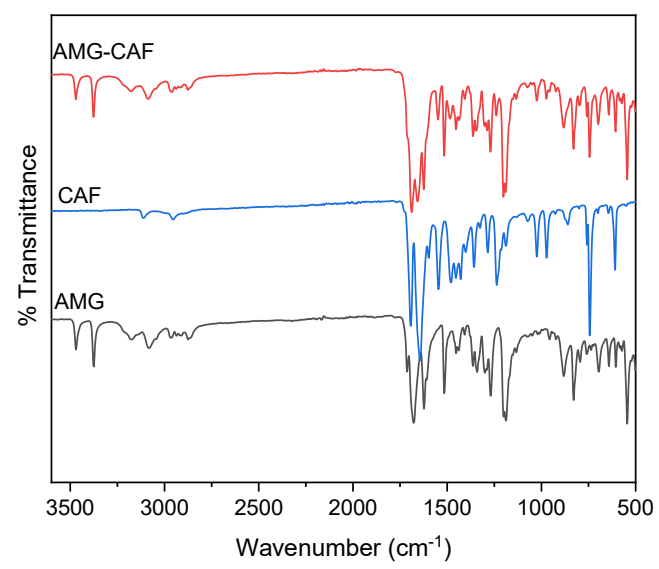

(a)

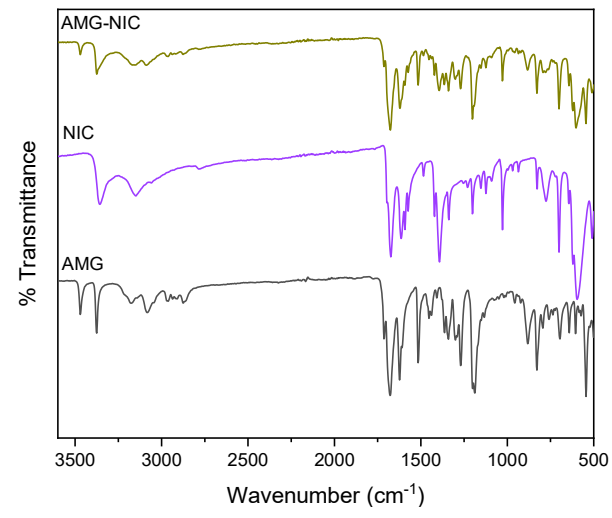

(b)

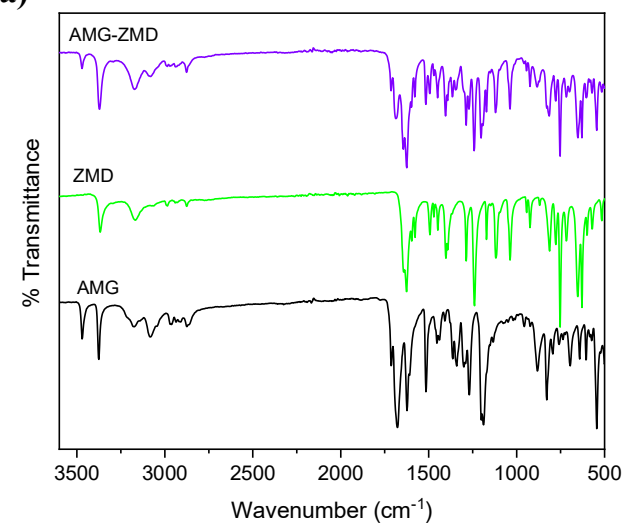

(c)

Figure 4 Comparison of FT-IR spectra of starting materials AMG and coformer with their respective eutectic composition for (a) AMG and CAF, (b) AMG and NIC, and (c) AMG and ZMD. 


\section{Scanning Electron Microscopy (SEM) Analysis}

The micrographs obtained from SEM are shown in Figure 5. SEM images accurately contrast the morphological characteristics of AMG and the coformers. AMG-CAF, AMG-ZMD and AMGNIC have significantly different morphologies compared to the parent components. A noteworthy reduction in particle size in the grinding process is also observed in the eutectic systems.

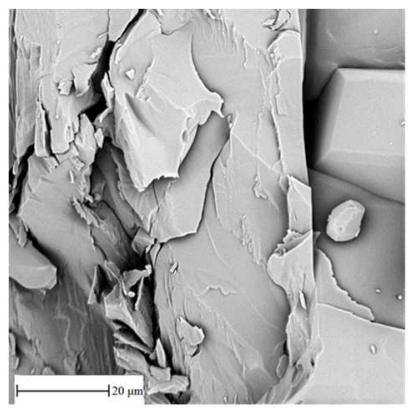

AMG

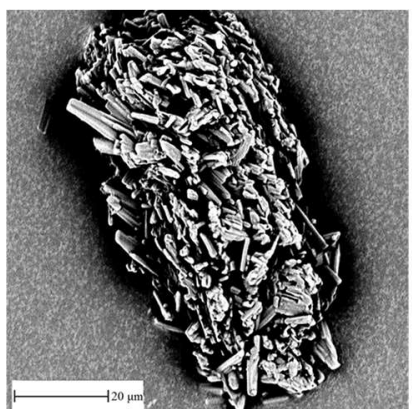

CAF

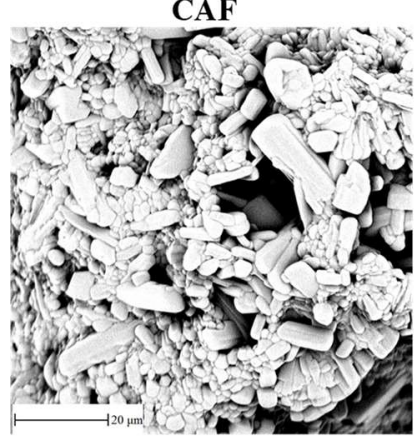

NIC

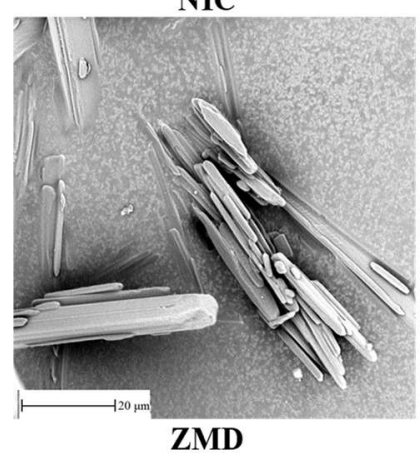

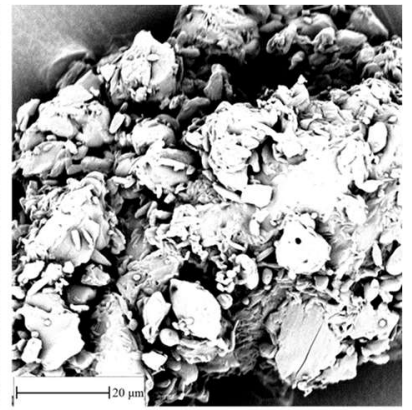

AMG-CAF

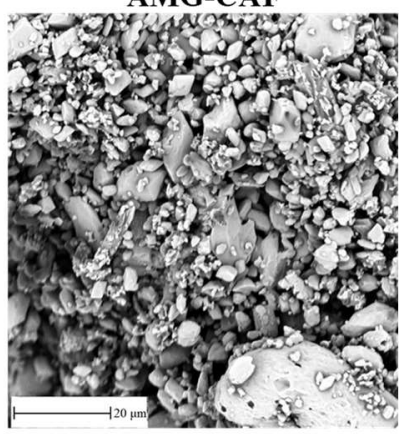

AMG-NIC

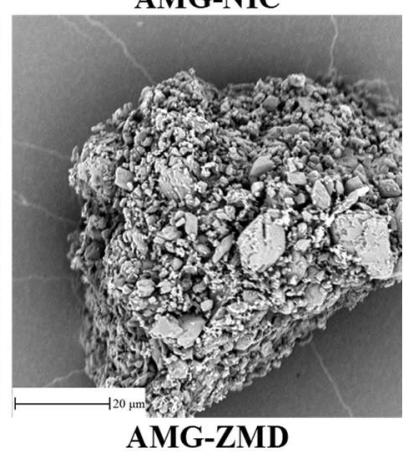

Figure 5 Comparison of micrographs of pure AMG and coformers with their respective eutectic mixtures at 200x magnification.

\section{Phase diagrams and related Tammann plots}

From the integration of the eutectic melting effects at different mixture compositions the related eutectic melting enthalpy, $\Delta \mathrm{H}_{\text {eut }}$ can be obtained. At the eutectic composition, the melting enthalpy 
is maximum and gradually decreases with composition towards the pure components. Therefore, plotting the $\Delta \mathrm{H}_{\text {eut }}$ values as a function of the composition (Tammann plot), the intersection of the two linearized parts provides a good measure of the eutectic composition in the respective binary system.

AMG-CAF system The presence of the single melting endotherm at $127.2{ }^{\circ} \mathrm{C}$ (see Figure $3 \mathrm{a}$ ), lower than that of both the starting components $\mathrm{AMG}$ and $\mathrm{CAF}$, indicated the formation of the eutectic composition in the AMG-CAF system at $75 \mathrm{wt}$ \% of AMG. The phase diagram and the Tammann plot for this system are presented in Figures $6 \mathrm{a}$ and $6 \mathrm{~b}$. In the phase diagram, the liquidus and solidus temperatures are plotted as a function of weight \% (wt.\%) of AMG in the CAF-AMG system. It shows the enantiotropic behavior of caffeine and specifies the polymorphic phase transition at $137.7^{\circ} \mathrm{C}$ confirming the literature data. ${ }^{25-26}$ Also it indicates the eutectic composition at $\sim 75$ wt. $\%$ what is verified in the Tammann plot (Figure $6 \mathrm{~b}$ ). In addition, the latter indicates a partial solid solution behavior of CAF in AMG (indicated by the dashed line in Figure 6b). However, since this is based on only two points, it needs to be verified what is hard to be done in the small existence region of AMG in the phase diagram. The value of partial miscibility of CAF in the AMG crystal lattice was found to be $\sim 95 \pm 2 \mathrm{wt} . \%$ of AMG.

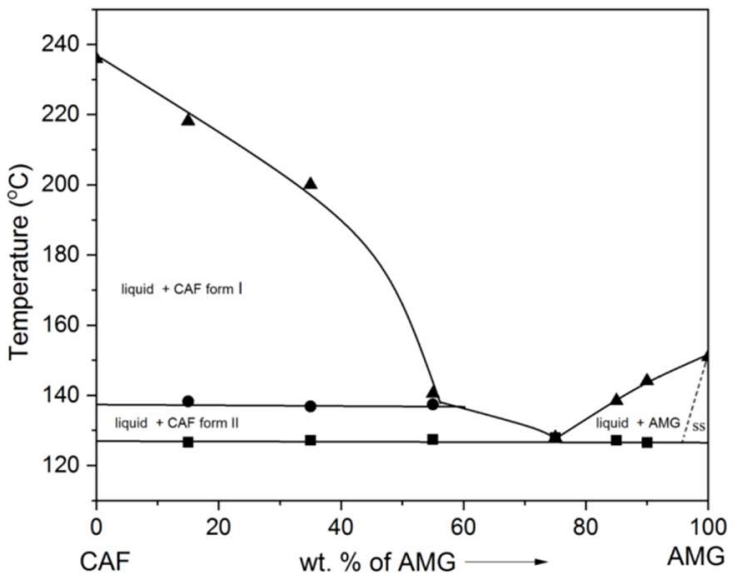

(a)

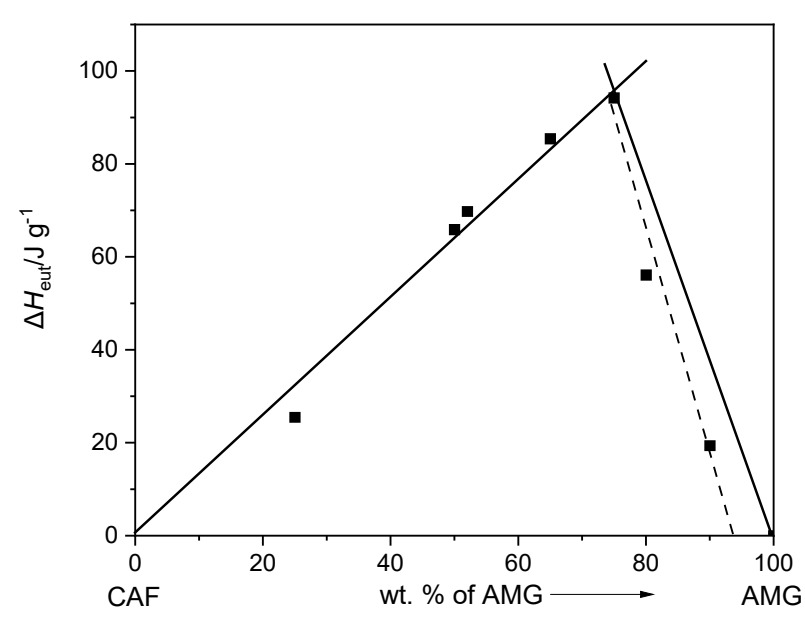

(b)

Figure 6 (a) Binary melt phase diagram and (b) Tammann plot of the AMG-CAF system. 
AMG-NIC system The DSC profiles in Figure 3b illustrate the melting behavior of solid samples at different AMG-NIC compositions prepared by neat grinding. The system shows simple eutectic behavior, thus mixtures of both components exhibit two endothermic peaks in DSC curves corresponding to eutectic melting and the subsequent dissolution effect of the excess compound. Figure 7a presents the derived melt phase diagram with a eutectic composition of $\sim 50 \mathrm{wt} \%$ AMG. The eutectic temperature in this system was found on average at $99.2^{\circ} \mathrm{C}$ (Table 1). The Tammann plot shown in Figure $7 \mathrm{~b}$ confirms the $\sim 50 \mathrm{wt} . \%$ eutectic composition.

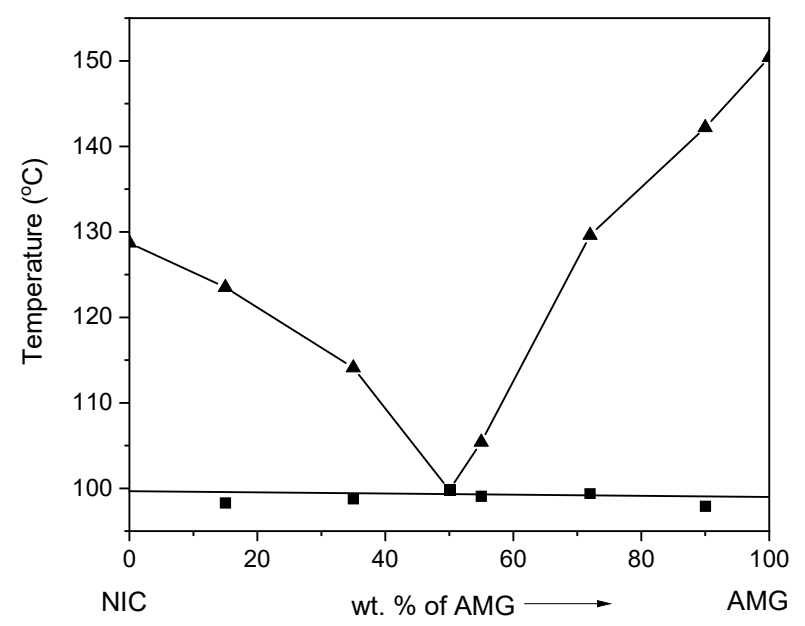

(a)

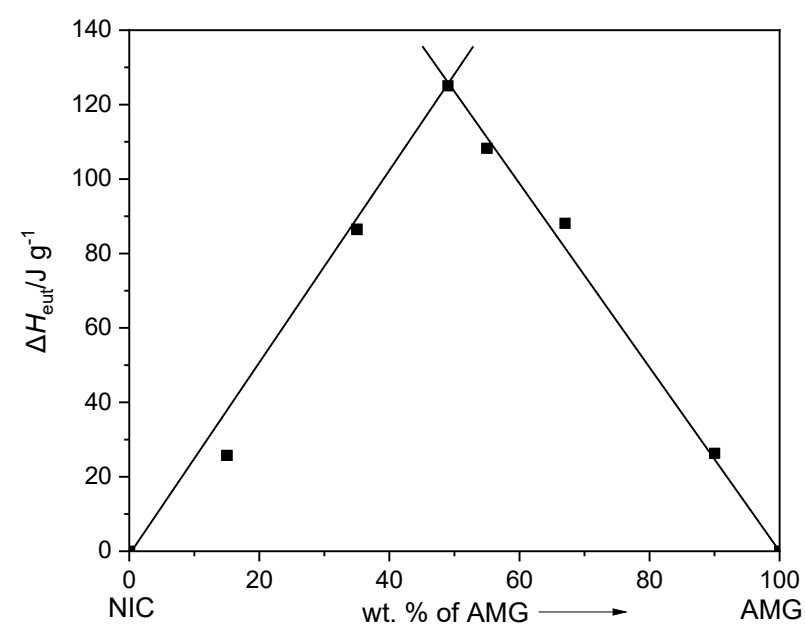

(b)

Figure 7 (a) Binary melt phase diagram and (b) Tammann plot of the AMG-NIC system.

AMG-ZMD system As for the AMG-NIC system, the melt phase diagram of the AMG-ZMD system is characterized by a simple eutectic, possessing a eutectic point at $101.8^{\circ} \mathrm{C}$ and composition of $\sim 50 \mathrm{wt} \%$ of AMG. The eutectic composition was derived from the single endothermic event in the DSC thermogram and also from the intersection of the ZMD and AMG liquidus curves. Tammann's plot (Figure 8b) for the AMG-ZMD system verifies the eutectic composition close to $50 \mathrm{wt}$ \% AMG and indicates partial miscibility at the solid-state close to the ZMD side (indicated by the dashed line in Figure $8 \mathrm{~b}$ ), but similar to the AMG-CAF system, need more detailed studies if important. 


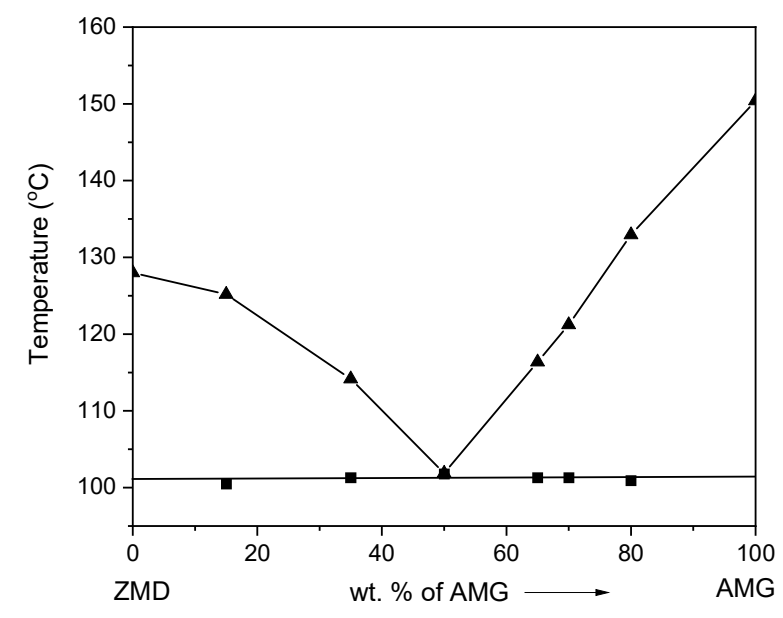

(a)

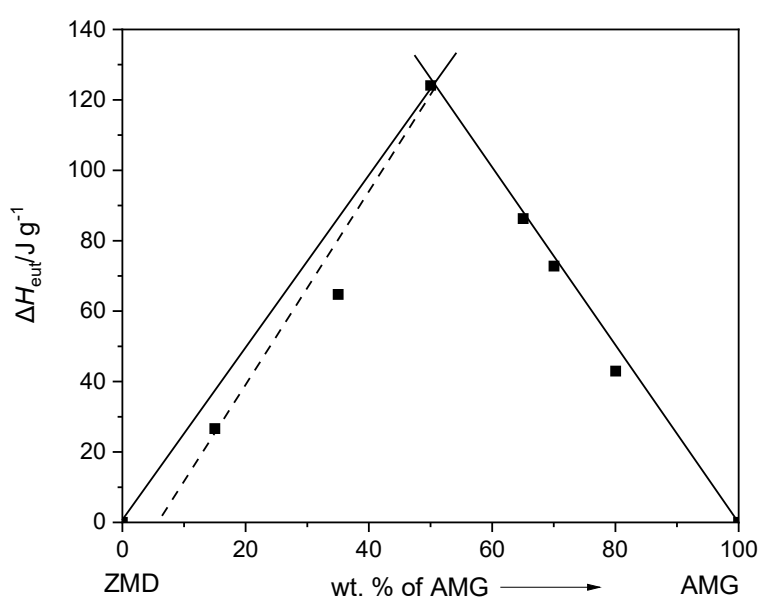

(b)

Figure 8 (a) Binary melt phase diagram and (b) Tammann plot of the AMG-ZMD system.

\section{Aqueous solubility of the eutectics}

To measure the solubility each experiment was performed in a jacketed, circulating flask maintained at $25{ }^{\circ} \mathrm{C}$ and in two sets to ensure consistency. Interestingly, the eutectics show significantly improved solubility over the parent drug AMG with a solubility of $1.9 \mathrm{mg} / \mathrm{mL}$ at $25^{\circ} \mathrm{C}$ (See Figure 9). The AMG-CAF, AMG-NIC and AMG-ZMD eutectics exhibit nearly 2.5fold and 1.5 times higher solubility than AMG.

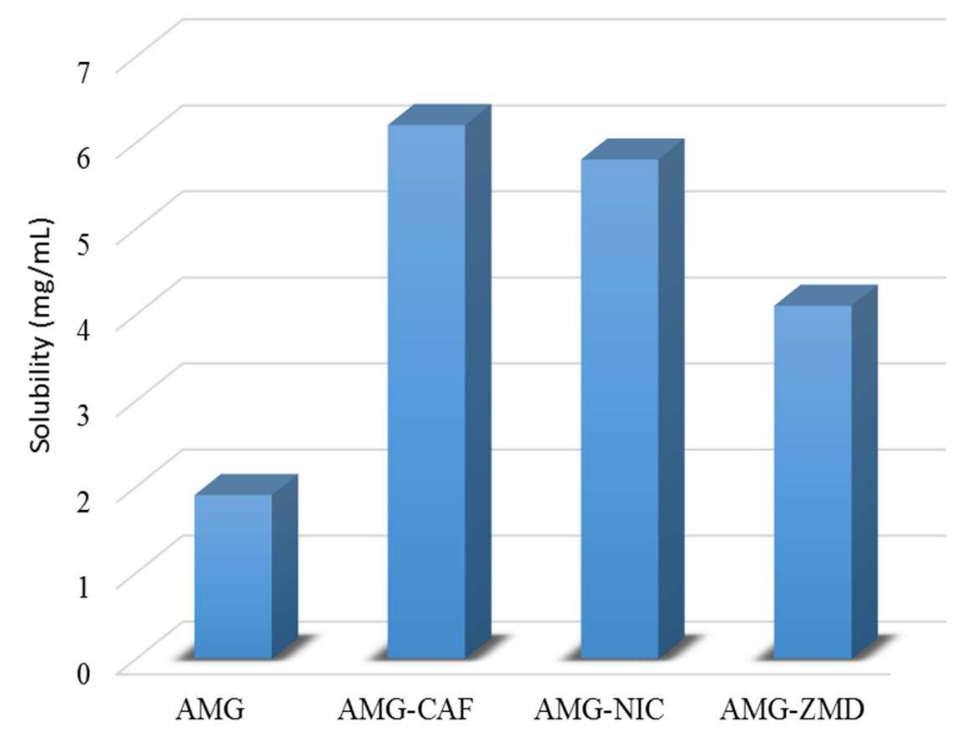

Figure 9 Comparison of solubilities of AMG and its eutectic mixtures in water at $25^{\circ} \mathrm{C}$. 
Different thermodynamic parameters like melting temperature depression and the heat of fusion $\left(\Delta \mathrm{H}_{\mathrm{f}}\right)$ can affect the overall solubility. ${ }^{27},{ }^{28}$ Considerable decreases in the $\Delta \mathrm{H}_{\mathrm{f}}$ is observed for AMGCAF (see Table 1) systems and expectedly it is showing higher solubility than AMG. However, higher $\Delta \mathrm{H}_{\mathrm{f}}$ has been observed for the eutectics AMG-ZMD and AMG-NIC, as a result, the solubility is the difference is not very significant. Particularly, despite having higher soluble coformer NIC, the eutectics AMG-NIC exhibited very little solubility. The experimental solubility result is found to be supported by the ideal solubility data for the eutectic mixtures calculated by using the van't Hoff equation.

\section{Conclusions}

Solvent-assisted mechanochemical grinding of the drug aminoglutethimide with pharmaceutical coformers enabled the production of binary eutectic mixtures. Considerable lowering of the melting points in the eutectic mixtures was determined from DSC analysis. Analogously, the aqueous solubility of the binary eutectic mixtures could be significantly improved compared to the pure AMG. Solubilities at $25^{\circ} \mathrm{C}$ were found to be 1.5-2.5 times higher than for AMG. The final aim of the work was to improve AMG solubility through physical form alteration, and this objective was achieved by the formation of binary eutectic mixtures with caffeine and nicotinamide as drug-drug eutectics.

\section{Acknowledgement}

We thank Stefanie Oberländer and Jacqueline Kaufmann for their help in PXRD and DSC measurements.

\section{References}

1. Cherukuvada, S.; Nangia, A., Eutectics as improved pharmaceutical materials: design, properties and characterization. Chem. Commun. 2014, 50 (8), 906-923.

2. Saikia, B.; Pathak, D.; Sarma, B., Variable stoichiometry cocrystals: occurrence and significance. CrystEngComm 2021, 23 (26), 4583-4606. 
3. Araya-Sibaja, A. M.; Vega-Baudrit, J. R.; Guillén-Girón, T.; Navarro-Hoyos, M.; Cuffini, S. L., Drug Solubility Enhancement through the Preparation of Multicomponent Organic Materials: Eutectics of Lovastatin with Carboxylic Acids. Pharmaceutics 2019, 11 (3), 112.

4. Bazzo, G. C.; Pezzini, B. R.; Stulzer, H. K., Eutectic mixtures as an approach to enhance solubility, dissolution rate and oral bioavailability of poorly water-soluble drugs. Int. J. Pharm. 2020, 588, 119741.

5. Moore, M. D.; Wildfong, P. L. D., Aqueous Solubility Enhancement Through Engineering of Binary Solid Composites: Pharmaceutical Applications. J. Pharm. Innov. 2009, 4 (1), 36-49.

6. Saikia, B.; Bora, P.; Khatioda, R.; Sarma, B., Hydrogen Bond Synthons in the Interplay of Solubility and Membrane Permeability/Diffusion in Variable Stoichiometry Drug Cocrystals. Cryst. Growth Des. 2015, 15 (11), 5593-5603.

7. Wünsche, S.; Yuan, L.; Seidel-Morgenstern, A.; Lorenz, H., A Contribution to the Solid State Forms of Bis(demethoxy)curcumin: Co-Crystal Screening and Characterization. Molecules 2021, $26(3), 720$.

8. Alhadid, A.; Mokrushina, L.; Minceva, M., Design of Deep Eutectic Systems: A Simple Approach for Preselecting Eutectic Mixture Constituents. Molecules 2020, 25 (5), 1077.

9. Gala, U.; Chuong, M. C.; Varanasi, R.; Chauhan, H., Characterization and Comparison of Lidocaine-Tetracaine and Lidocaine-Camphor Eutectic Mixtures Based on Their Crystallization and Hydrogen-Bonding Abilities. AAPS PharmSciTech 2015, 16 (3), 528-536.

10. Cherukuvada, S.; Guru Row, T. N., Comprehending the Formation of Eutectics and Cocrystals in Terms of Design and Their Structural Interrelationships. Cryst. Growth Des. 2014, 14 (8), 4187-4198.

11. Stoler, E.; Warner, J. C., Non-Covalent Derivatives: Cocrystals and Eutectics. Molecules 2015, 20 (8), 14833-14848.

12. Hyun, S.-M.; Lee, B. J.; Abuzar, S. M.; Lee, S.; Joo, Y.; Hong, S.-H.; Kang, H.; Kwon, K.A.; Velaga, S.; Hwang, S.-J., Preparation, characterization, and evaluation of celecoxib eutectic mixtures with adipic acid/saccharin for improvement of wettability and dissolution rate. Int. J. Pharm. 2019, 554, 61-71.

13. Vasconcelos, T.; Sarmento, B.; Costa, P., Solid dispersions as strategy to improve oral bioavailability of poor water soluble drugs. Drug Discov. 2007, 12 (23), 1068-1075. 
14. Goud, N. R.; Suresh, K.; Sanphui, P.; Nangia, A., Fast dissolving eutectic compositions of curcumin. Int. J. Pharm. 2012, 439 (1), 63-72.

15. Bi, M.; Hwang, S.-J.; Morris, K. R., Mechanism of eutectic formation upon compaction and its effects on tablet properties. Thermochim. Acta 2003, 404 (1), 213-226.

16. Zalac, S.; Khan, M. Z. I.; Gabelica, V.; Tudja, M.; Mestrovic, E.; Romih, M., ParacetamolPropyphenazone Interaction and Formulation Difficulties Associated with Eutectic Formation in Combination Solid Dosage Forms. Chem. Pharm. Bull. 1999, 47 (3), 302-307.

17. Harris, A. L.; Dowsett, M.; Stuart-Harris, R.; Smith, I. E., Role of aminoglutethimide in male breast cancer. Br. J. Cancer 1987, 54 (4), 657-660.

18. Santen, R. J.; Misbin, R. I., Aminoglutethimide: Review of Pharmacology and Clinical Use. Pharmacotherapy: The Journal of Human Pharmacology and Drug Therapy 1981, 1 (2), 95-119.

19. Manni, A., Endocrine therapy of metastatic breast cancer. J. Endocrinol. Invest. 1989, 12 (5), 357-372.

20. Dahan, A.; Miller, J. M.; Amidon, G. L., Prediction of solubility and permeability class membership: provisional BCS classification of the world's top oral drugs. The AAPS Journal 2009, $11(4), 740-6$.

21. Faudone, G.; Arifi, S.; Merk, D., The Medicinal Chemistry of Caffeine. J. Med. Chem. 2021, 64 (11), 7156-7178.

22. Gehring, W., Nicotinic acid/niacinamide and the skin. J. Cosmet. Dermatol. 2004, 3 (2), 8893.

23. Aitipamula, S.; Chow, P. S.; Tan, R. B. H., Trimorphs of a pharmaceutical cocrystal involving two active pharmaceutical ingredients: potential relevance to combination drugs. CrystEngComm 2009, 11 (9), 1823-1827.

24. Macrae, C. F.; Sovago, I.; Cottrell, S. J.; Galek, P. T. A.; McCabe, P.; Pidcock, E.; Platings, M.; Shields, G. P.; Stevens, J. S.; Towler, M.; Wood, P. A., Mercury 4.0: from visualization to analysis, design and prediction. J. Appl. Crystallogr. 2020, 53 (1), 226-235.

25. Dichi, E.; Sghaier, M.; Guiblin, N., Reinvestigation of the paracetamol-caffeine, aspirincaffeine, and paracetamol-aspirin phase equilibria diagrams. J. Therm. Anal. Calorim. 2018, 131 (3), 2141-2155.

26. Dichi, E.; Legendre, B.; Sghaier, M., Physico-chemical characterisation of a new polymorph of caffeine. J. Therm. Anal. Calorim. 2014, 115 (2), 1551-1561. 
27. Lorenz, H., Solubility and Solution Equilibria in Crystallization. In Crystallization: Basic Concepts and Industrial Applications, Beckmann, D. W., Ed. Wiley-VCH: Weinheim, 2013; pp. $35-74$.

28. Tao, M.; Wang, Z.; Gong, J.; Hao, H.; Wang, J., Determination of the Solubility, Dissolution Enthalpy, and Entropy of Pioglitazone Hydrochloride (Form II) in Different Pure Solvents. Ind. Eng. Chem. Res 2013, 52 (8), 3036-3041. 